\title{
La Psicología en la prevención y manejo del COVID-19. Aportes desde la evidencia inicial
}

\section{Psychology in the prevention and management of COVID-19. Contributions from the initial evidence}

\author{
Alfonso Urzúa \\ Escuela de Psicología, Universidad Católica del Norte, Chile \\ Pablo Vera-Villarroel \\ Centro Analysis I+D, Chile \\ Alejandra Caqueo-Urízar \\ Instituto de Alta Investigación, Universidad de Tarapacá, Chile \\ Roberto Polanco-Carrasco \\ Cuadernos de Neuropsicología / Panamerican Journal of Neuropsychology, Chile \\ Rec (30 de Marzo de 2020) Acept (12 de Abril de 2020)
}

\begin{abstract}
Resumen
En diciembre de 2019, se informaron casos de neumonía potencialmente mortal en Wuhan, provincia de Hubei, China (COVID-19). Esta enfermedad se ha extendido rápidamente por todo el mundo con miles de casos confirmados y muertes, transformándose en pandemia y desafiado los sistemas de salud pública. No existe aún vacuna ni tratamiento científicamente probado, sin embargo, se ha identificado los comportamientos exactos que pueden evitar el contagio y propagación. El presente artículo sistematiza información disponible inicial sobre psicología y COVID-19. Se discute que gran parte del problema de la enfermedad se puede evitar cambiando los comportamientos de las personas y que la psicología puede ayudar a explicar, prevenir e intervenir para su solución. La psicología cuenta con evidencia científica disponible que explica todos estos fenómenos, evidencia que debe ser puesta en relieve por los mismos actores de las disciplinas a disposición de otras áreas del conocimiento y sobre todo para los tomadores de decisión.
\end{abstract}

Palabras Clave: Corona-Virus, COVID-19, Psicología de la Salud, Salud Publica

\begin{abstract}
In December 2019, highly lethal cases of pneumonia were reported in Wuhan, Hubei Province, China (COVID-19). This disease has spread rapidly around the world with thousands of confirmed cases and deaths, becoming a pandemic and challenging public health systems. There is no vaccine or scientifically proven treatment yet, but the exact behaviours that can prevent transmission and spread have been identified. This article systematizes initial available information on psychology and COVID-19. It is discussed that much of the problem of the disease can be avoided by changing people's behaviors and that psychology can help explain, prevent and intervene to solve it. Psychology has scientific evidence available that explains all these phenomena, evidence that should be highlighted by the same actors in the disciplines available to other areas of knowledge and especially for decision makers.

Key words: Corona-Virus, COVID-19, Health Psychology, Public Health
\end{abstract}

Correspondencia: Dr. Alfonso Urzúa. Avda. Angamos 0610, Antofagasta, Chile. Email. alurzua@ucn.cl 


\section{Introducción}

En diciembre de 2019, se informaron casos de neumonía potencialmente mortal en Wuhan, provincia de Hubei, China. A enero de 2020, esta enfermedad denominada COVID-19 se había extendido a 19 países con 11.791 casos confirmados, incluyendo 213 muertes (Poudel et al., 2020). Los síntomas informados incluyen fiebre, tos, fatiga, neumonía, dolor de cabeza, diarrea, hemoptisis y disnea. Pese a la baja gravedad de los síntomas, el contagio es muy rápido, lo que hace que, aunque sea proporcionalmente baja la cantidad de casos que podrían llegar a ser graves o letales, el fenómeno de rápida propagación puede colapsar los sistemas sanitarios que proporcionan ayuda a los pacientes más graves. Adicionalmente, si llega el virus a personas con otras enfermedades o a adultos mayores, la enfermedad se transforme en grave y, por ende, urgente la atención adecuada de esos casos (Guo, Cao, Hong et al., 2020).

Un análisis simple de estos hechos muestra que, a efectos de la salud pública, la gravedad no es únicamente el virus en sí mismo, sino la rapidez de contagio a la población y la llegada a los grupos de riesgo en poco tiempo, lo que conlleva a una inadecuada capacidad de respuesta de los servicios de urgencia ante una alta demanda en poco tiempo.

Entonces, ¿qué variables hacen que un virus /enfermedad pueda cerrar fronteras y colapsar sanitaria, política y económicamente a todo el planeta? ¿Qué factores más allá de lo biológico y de la ausencia aun de una vacuna hacen que se transforme en uno de los peores fenómenos en salud de los últimos años en todo el mundo? Creemos que la respuesta es fundamentalmente el comportamiento de las personas.

Hace años, Bayes y Ribes argumentaban cómo la psicología estaba involucrada en una enfermedad importante y menos conocida en ese momento como es el VIH-SIDA (Bayes \& Ribes, 1992). Apelando a la evidencia y a modelos conceptuales y teóricos de la psicología experimental, su tesis principal era que, independientemente del origen del virus y su potencial tratamiento médico y /o la generación de una vacuna en el futuro, su adquisición proceso y tratamiento era desde un inicio y final un proceso psicológico. La investigación posterior ha generado abundante evidencia que muestra que tanto la prevención, la adherencia al tratamiento o el enfrentamiento y avance de múltiples enfermedades son un tema de comportamiento.

Diversos modelos psicológicos tales como el de creencias en salud, transteórico, locus de control, autoeficacia, acción razonada, entre otros, intentan explicar cómo adquirimos y mantenemos las conductas en salud (Vera-Villarroel, Livacic-Rojas, Fernández \& Oblitas, 2015). La evidencia muestra que los mecanismos por los cuales adquirimos, mantenemos y eliminamos nuestros comportamientos son principalmente por medio del aprendizaje, por ende, los comportamientos involucrados en los procesos de salud y enfermedad responden a los mismos mecanismos. De esta forma, el pasar de una condición de ausencia de enfermedad a estar enfermo e incluso el éxito o no del tratamiento estará determinado por nuestras creencias (cogniciones), emociones (como predisposiciones a la acción) y finalmente nuestro comportamiento pro o riesgoso para la salud.

En el caso de la pandemia generada por el COVID-19, la evidencia ha mostrado que, independiente de la etapa de exposición al virus en que pueda estar una persona, ya sea en cuarentena, al haber estado expuesta, en aislamiento, al ya estar infectada, bajo una situación preventiva de distanciamiento social, o bien post alta del virus, todas las medidas preventivas sugeridas incorporan medidas comportamentales.

En este contexto, la psicología como la ciencia experta en comportamiento puede aportar evidencia para que los tomadores de decisiones (gerentes directores, ministros políticos) la incorporen en los diagnósticos, planificaciones e intervenciones públicas y privadas. En este contexto, presentamos una revisión de hallazgos recientes desde la psicología que pueden aportar el enfrentamiento del virus.

\section{Evidencia cientifica psicológica previa de enfermedades similares}

El Covid-19 es probablemente la infección respiratoria que ha causado más impacto y daño internacional en las últimas décadas, sin embargo, han existido enfermedades similares previas recientes como el Síndrome Respiratorio del Medio Oriente (MERS por sus siglas en inglés) y el Síndrome respiratorio agudo severo (SARS por sus siglas en inglés), de las que podemos extraer evidencia importante para entender mejor el Covid-19 y mejor aún, intentar sacar conclusiones que permitan predecir las demandas para la salud en general y la psicología en particular. 
El MERS fue identificado en su momento como una nueva infección respiratoria en el año 2012 en Arabia Saudita (Zaki, van Boheemen, Bestebroer, Osterhaus \& Fouchier, 2012) y en Corea el 2015 caracterizándose también por su rápido contagio y muertes. En plena epidemia, Parka, Leeb, Parkc \& Choid (2018) realizaron un estudio en enfermeras hospitalarias, encontrando que tanto el estigma como la resistencia — conceptualizada como disposición de la personalidad caracterizada por un involucramiento en lo que están haciendo (compromiso), en que creen y actúan como si pudieran influenciar los eventos que forman sus vidas (control), y consideran que el cambio no sólo es normal sino también un estímulo para el desarrollo (desafío) - ejercen un efecto directo sobre la salud mental, el cual podría ser parcialmente mediado por la presencia de estrés, concluyendo sobre la necesidad de implementar aplicaciones en salud mental mediante un programa de educación/intervención que considere estos aspectos.

En Corea del Sur el MERS fue considerado como una gran amenaza para la salud pública, donde la gravedad se vio aumentada por las respuestas inapropiadas de las principales instituciones y la comunidad. Yang \& Cho (2017) analizaron las posibles fuentes de confusión durante el brote de MERS y los factores que podían afectar el comportamiento de las personas, reportando que la percepción del riesgo fue clave en las reacciones de las personas y se relacionó con la mayoría de los factores estudiados y principalmente con la reacción exagerada del público. Sin embargo, el conocimiento sobre la naturaleza de la enfermedad disminuyo la reacción exagerada y mejoró el cumplimiento de las instrucciones de la cuarentena. De esta forma, los autores concluyen la importancia de proporcionar adecuadamente información sobre la enfermedad y de entregar datos precisos al público y establecer confianza y facilitar el desarrollo de una actitud será importante en futuras crisis similares de forma de garantizar una respuesta rápida a los brotes de enfermedades y para prevenir comportamientos inadecuados.

En Corea igualmente se estudió el efecto del MERS en la salud mental de personas que estuvieron en cuarentena por haber estado expuestas al virus, en un periodo de 4 a 6 meses después de haber concluido la cuarentena, encontrando como factores de riesgo para experimentar sintomatología ansiosa y sentimientos de rabia la presencia de síntomas relacionados con el MERS durante el aislamiento, suministros inadecuados (comida, ropa, alojamiento), actividades de redes sociales (correo electrónico, texto, Internet), historial de enfermedades psiquiátricas y pérdidas financieras, concluyendo que estos problemas de salud mental pueden prevenirse al proporcionar apoyo de salud mental a las personas con salud mental vulnerable, así como proporcionando información precisa y suministros apropiados (Jeong et al., 2016)

El 2003, el mundo se vio afectado por la mortal epidemia de SARS. Dadas las exigencias y demandas de esta enfermedad tanto para la comunidad como para el personal de la salud se analizaron las posibles consecuencias tiempo después. Lee et al., (2007) analizaron las consecuencias psicológicas en sobrevivientes trabajadores sanitarios y no sanitarios un año después del brote de la enfermedad. Los resultados un año después del brote mostraron que los sobrevivientes de SARS todavía tenían niveles elevados de estrés y niveles preocupantes de angustia psicológica y específicamente del SARS del trabajador de la salud fue significativamente mayor, lo que sugiere en la necesidad de establecer programas de apoyo e intervención posterior y específicos para los profesionales de la Salud. En China, se estudiaron las relaciones entre los estresores y el estilo de afrontamiento utilizado frente al SARS con el ajuste psicológico, en estudiantes universitarios, encontrando que la presencia de estresantes y un estilo de afrontamiento evitativo predijeron la presencia de sintomatología, en tanto un afrontamiento de tipo activo predijo una mayor satisfacción con la vida al controlar los estresores (Main, Zhou, Ma et al., 2011).

\section{Impacto psicológico del COVID-19}

Debido a lo reciente de la enfermedad se cuenta con poca evidencia disponible, sin embargo, ya especialistas reportan una serie de reacciones y estados psicológicos observados en la población, incluyendo efectos en la salud mental (Torales, O’Higgins, Castaldelli-Maia \& Ventriglio, 2020). Así, Shigemura, Ursano, Morganstein, Kurosawa \& Benedek, (2020) informan que las primeras respuestas emocionales de las personas incluyen miedo e incertidumbre extremos. Por otra parte, y dado el miedo y percepciones distorsionadas de riesgo y consecuencias dañinas se informa de comportamientos sociales negativos. Estos estados y conductas pueden dar pie a problemas en salud mental publica incluidas reacciones de angustia (insomnio, ira, miedo extremo a la enfermedad incluso en personas no expuestas), y de comportamientos riesgosos para la salud como mayor uso de alcohol y tabaco y aislamiento social), trastornos específicos como trastorno de estrés postraumático, 
trastornos de ansiedad, depresión, y somatizaciones. Estos últimos síntomas y la necesidad de intervenciones terapéuticas son igualmente recomendables para niños (She, Yanq, Wang et al., 2020). Un efecto similar lo reportan Li, Wan, Xue, Zhao y Zhu (2020), quienes estudian los mensajes enviados de Weibo (sitio web chino de redes sociales) antes y después de la declaración del COVID-19 el 20 de enero del 2020, encontrando que las emociones negativas como la ansiedad, la depresión y la indignación, así como la sensibilidad a los riesgos sociales aumentaron, en tanto disminuyeron las emociones positivas (por ejemplo, la felicidad) y la satisfacción con la vida. En general encontraron que las personas se preocupaban más por su salud y su familia, y menos por el ocio y amigos.

Por otra parte, entre el 31 de enero al 2 de febrero de 2020 se realizó una encuesta en línea a 1210 personas de varias ciudades chinas, encontrándose que el $53.8 \%$ de los encuestados calificó el impacto psicológico de la enfermedad como moderado o severo; $16.5 \%$ reportaron síntomas depresivos moderados a severos; un $28.8 \%$ reportó síntomas de ansiedad moderada a severa; y $8.1 \%$ informaron niveles de estrés moderados a severos. La mayoría de los encuestados informó pasar de 20 a 24 h por día en casa (84.7\%) y además un 75.2 presentaba preocupación de que sus familiares se contagiaran de la enfermedad. Un aspecto importante para considerar en función de incorporar para intervenciones futuras es que se encontró que la información específica y actualizada sobre la salud (p. Ej., tratamiento, situación de brote local) y medidas de precaución específicas como higiene de manos y uso de una mascarilla) se asociaron con un menor impacto psicológico del brote y menores niveles de estrés, ansiedad, y depresión (Wang, Pan, Wan et al., 2020)

Con respecto a las intervenciones psicológicas, obviamente a estas alturas es escasa la información que se puede considerar. Aun así, ya se cuenta con alguna información disponible para la comunidad científica. En este sentido, los especialistas concuerdan que las intervenciones en crisis deberían ser consideradas como una medida a implementar en todos los grupos afectados, ya sea pacientes personales médico, contactos cercanos, personas en las áreas afectadas, así como público en general (Zhu, Chen, Ji, et al., 2020). Las intervenciones psicológicas en crisis tienen como objetivo minimizar los daños psicológicos y proveer asistencia durante la prevención y el control de la epidemia, intentando evitar así problemas posteriores como el estrés post traumático.

Dado que los recursos son limitados, Jiang et al. (2020), con base a los protocolos establecidos por el Gobierno Chino y aplicados en Shangai en pacientes con neumonía por coronavirus, reporta cuatro niveles de atención que podían ayudar a la priorización de la atención: 1) pacientes con síntomas severos, personal médico de choque investigadores y personal administrativo, 2) pacientes con sintomatología mediana, contactos cercanos, pacientes sospechosos o que ingresan con fiebre para tratamiento, 3) personas relacionadas con los grupos 1 y 2, como miembros de la familia, colegas o amigos, rescatistas, 4) personas en áreas afectadas, grupos vulnerables o público en general.

La cuarentena, concebida como la separación y restricción del movimiento de personas que han sido potencialmente expuestas al virus, también ha sido un factor estudiado. Brooks et al (2020), realizaron una revisión rápida acerca del impacto psicológico de la cuarentena y cómo reducirlo. Los autores plantean que se han reportado efectos psicológicos negativos, como síntomas de estrés postraumático, confusión e ira. Igualmente, reportan que existirían diversos factores que incidirían en la respuesta de las personas al distanciamiento social, como por ejemplo el tiempo de duración de la cuarentena, temores de infección, frustración, aburrimiento, suministros inadecuados, información inadecuada, pérdidas financieras y estigma. Plantean que en las situaciones en que la cuarentena se considera necesaria, no se debe poner a las personas en cuarentena por un período superior al requerido, debiéndosele proporcionar una justificación clara de la cuarentena e información sobre los protocolos, asegurando que se proporcionen suficientes suministros.

\section{Impacto Psicológico en trabajadores de la Salud}

Desde el brote de la enfermedad muchos trabajadores de la Salud han sido infectados con el virus. Dai, Hu, Xiong, Qiu \& Yuan (2020). investigaron la percepción de riesgo y el estado psicológico inmediato de trabajadores de la Salud en la etapa temprana de la epidemia de COVID-19. Se evaluó entonces la percepción del riesgo y el estado psicológico junto a características demográficas y las experiencias de exposición a COVID-19. Se encuestaron a 4.357 sujetos. Las principales preocupaciones de los trabajadores de la salud fueron en primer lugar infección de colegas (72.5\%), en segundo lugar, la infección de miembros de la familia (63.9\%), tercer lugar medidas de protección (52.3\%) y finalmente la violencia médica (48.5\%). Por otra parte, un 39\% de los 
trabajadores sanitarios presentaba problemas psicológicos, principalmente los que ya habían pasado por aislamiento y contagio a familiares o colegas.

\section{Intervenciones psicológicas en COVID-19}

En la dinámica de la enfermedad del Covid-19 se aprecia la relevancia de las conductas de las personas. La importancia de la identificación del comportamiento nos solo hace una comprensión más adecuada de la enfermedad, sino que permite diseñar, planificar las intervenciones más efectivas permitiendo en este caso a la Psicología poder aportar con todo su conocimiento científico de la Ciencia del comportamiento para el abordaje de esta Pandemia. En la figura 1. Se muestra un encadenamiento de comportamientos vinculadas a la fase de la enfermedad, desde los que todas las personas deben hacer hasta el momento de detección de la enfermedad (Poudel et al., 2020). De esta forma, ya se sabe cuáles serían las conductas preventivas; prácticas de higiene de manos, evitar el contacto público, uso de máscaras, detección de casos, localización de contactos, cuarentenas para reducir la transmisión. Ante la conducta de sospecha de presentar la enfermedad, primeros síntomas o el haber estado cerca de personas infectadas: acudir a centros de salud y realización de diagnóstico. Por último, si se ha detectado la presencia del virus en las personas comienza la realización del tratamiento que está vinculado nuevamente a conductas delimitadas y específicas como reposo y aislamiento y el tratamiento de los síntomas siguiendo las indicaciones médicas.

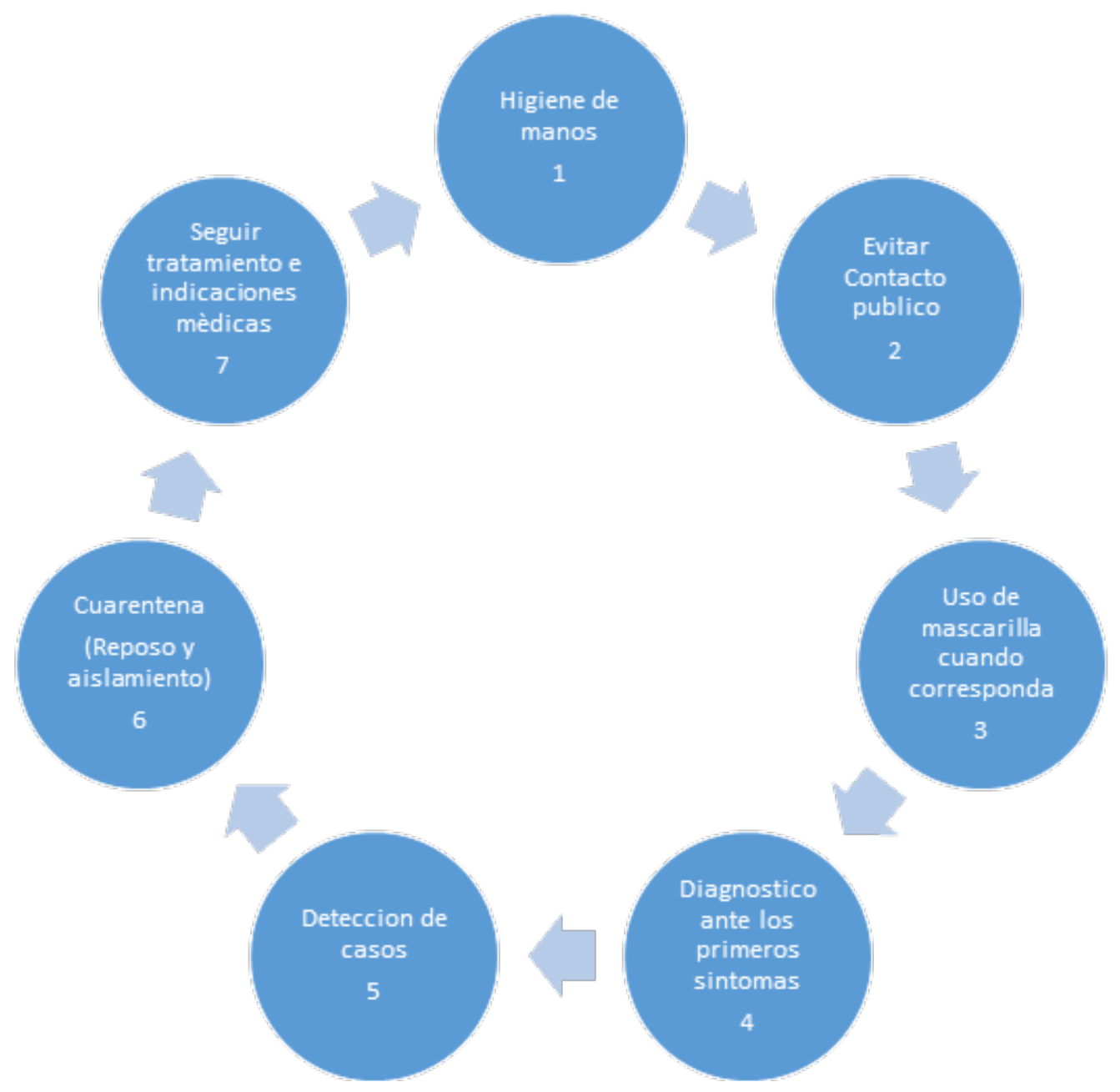

Figura 1. Mapa conductual del COVID-19. Adaptación de Poudel et al.2020 
Lunn, Belton, Lavin et al. (2020) proponen siete áreas en que la ciencia del comportamiento puede contribuir a frenar la propagación del virus en distintos niveles, no sólo por las autoridades nacionales y locales, sino también por organizaciones, lugares de trabajo e incluso hogares. Estas áreas son el lavado de manos, el contacto con la cara, el aislamiento, el comportamiento en público, los comportamientos indeseables (ej. xenofobia), la comunicación de crisis y las percepciones de riesgo. Estos investigadores plantean, por ejemplo, que la evidencia muestra que la educación y la información sobre la higiene de las manos no es suficiente, que el aislamiento puede causar distrés y problemas de salud mental que requieren atención o que las percepciones de riesgo pueden estar sesgadas o distorsionadas.

Con el fin de poder sistematizar la diversidad de áreas en que la psicología en tanto ciencia y profesión pueden contribuir al abordaje del COVID-19, Murphy (2020) desarrolló un marco que organiza los distintos aportes de la psicología, el cual hemos adaptado y organizado según los diferentes niveles de prevención en Salud Pública (Tablas 1 y 2.)

Tabla 1. Marco para la contribución de la psicología y el psicólogo a la pandemia de COVID-19 en la salud pública. Aspectos relacionados con la enfermedad.

\begin{tabular}{|c|c|c|c|}
\hline & Prevención Primaria & Prevención Secundaria & Prevención terciaria \\
\hline \multirow[t]{3}{*}{$\begin{array}{l}\text { 1.1 Conductas } \\
\text { de prevención }\end{array}$} & $\begin{array}{l}\text { Intervención con la finalidad de } \\
\text { reducir o eliminar el riesgo. Se } \\
\text { implementa antes de que haya } \\
\text { evidencia o sintomatología } \\
\text { de una enfermedad, afección, } \\
\text { daño o lesión. }\end{array}$ & $\begin{array}{l}\text { Tiene por objeto prevenir la } \\
\text { enfermedad/condición si ya ha } \\
\text { habido exposición a los factores } \\
\text { de riesgo y existe un potencial } \\
\text { desarrollo de la enfermedad o } \\
\text { condición, pero aún en la etapa } \\
\text { preclínica temprana o asin- } \\
\text { tomática }\end{array}$ & $\begin{array}{l}\text { Intervención implementada } \\
\text { cuando ya existe una enferme- } \\
\text { dad, lesión o sintomatología, } \\
\text { con el fin de retrasar o prevenir } \\
\text { su progreso o bien con fines de } \\
\text { rehabilitación }\end{array}$ \\
\hline & $\begin{array}{l}\text { Psicoeducación para la adop- } \\
\text { ción de conductas preventivas: } \\
\text { lavado de manos, distancia- } \\
\text { miento social }\end{array}$ & $\begin{array}{l}\text { Psicoeducación para la adopción } \\
\text { de conductas preventivas: lava- } \\
\text { do de manos, uso de mascara, } \\
\text { cuarentena }\end{array}$ & $\begin{array}{l}\text { Psicoeducación para la adop- } \\
\text { ción de conductas preventivas: } \\
\text { lavado de manos, uso de mas- } \\
\text { cara, aislamiento o cuarentena. }\end{array}$ \\
\hline & $\begin{array}{l}\text { Desarrollo de material educati- } \\
\text { vo con medidas preventivas }\end{array}$ & $\begin{array}{l}\text { Desarrollo de material educativo } \\
\text { con medidas preventivas }\end{array}$ & $\begin{array}{l}\text { Desarrollo de material educati- } \\
\text { vo con medidas preventivas }\end{array}$ \\
\hline
\end{tabular}

Apoyo información/comunicación pública

Entrenamiento en búsqueda racional de información

Manejo de creencias irracionales con relación a la enfermedad

Aumento percepción de vulnerabilidad

Desarrollo estrategias de afrontamiento

Entrenamiento en búsqueda racional de información

Manejo de creencias irracionales con relación a la enfermedad

Aumento percepción de vulnerabilidad

Desarrollo estrategias de afrontamiento

Optimismo ilusorio

1.2 Manejo de

Control de la ansiedad

Gestión de riesgo

síntomas psicológicos
Control de preocupación por cercanos

Desarrollo de tolerancia ante la incertidumbre
Control de la ansiedad

Gestión de riesgo

Manejo de la culpa

Control de preocupación por cercanos

Desarrollo de tolerancia ante la incertidumbre
Desarrollo estrategias de afrontamiento

Control de la ansiedad

Manejo de la culpa

Control de preocupación por cercanos 
Polanco-Carrasco

\begin{tabular}{lll}
\hline & Prevención Primaria & Prevención Secundaria \\
\hline $\begin{array}{l}\text { 1.3 Búsqueda } \\
\text { de ayuda }\end{array}$ & Interpretación/ percepción de & Interpretación/ percepción de \\
& síntomas (no hiper o hipo) & síntomas \\
& Percepción de riesgo & Percepción de riesgo \\
& Promover conductas de & Promover conductas de búsque- \\
& búsqueda de ayuda apropiadas & da de ayuda apropiadas
\end{tabular}

1.4. Cuidado psicológico de pacientes y familiares de pacientes
Promover el cuidado psicológico de pacientes internados o ambulatorios.

Promover el cuidado psicológico de pacientes internados o ambulatorios.
Prevención terciaria

Promover conductas de búsqueda de ayuda apropiadas

Promover el cuidado psicológico de pacientes internados o ambulatorios.
Desarrollo de intervenciones terapéuticas no presenciales

Manejo de complicaciones psicológicas: depresión, estrés, ansiedad, EPT

\section{Desarrollo de intervenciones terapéuticas no presenciales \\ Manejo de complicaciones psicológicas: depresión, estrés, ansiedad, EPT}

Apoyo psicológico a familia en casos de cuarentena
Desarrollo de intervenciones terapéuticas no presenciales/ presenciales

Manejo de complicaciones psicológicas: depresión, estrés, ansiedad, EPT

Apoyo terapéutico post alta Apoyo psicológico a familia en casos de aislamento u hospitalización

Apoyo en proceso de duelo a familiares

Tabla 2. Marco para la contribución de la psicología y el psicólogo a la pandemia de COVID-19 en la salud pública. Impacto profesional y social.

\begin{tabular}{ll}
\hline & Prevención Primaria \\
\hline 2.1 Ajuste en & Uso efectivo de servicios onl \\
la entrega de & Asesoría y consejería por \\
prestaciones & medios no presenciales \\
psicológicas & \\
& $\begin{array}{l}\text { Toma de decisiones/ manejo } \\
\text { del riesgo balanceando el } \\
\text { distanciamiento social y la } \\
\text { necesidad de consulta cara a } \\
\end{array}$ \\
& cara
\end{tabular}

2.2 Efecto del aislamiento y distanciamiento social
Mitigación de efectos de aislamiento Manejo del tiempo Promover actividad física Manejo de estrés en relaciones Prevención VIF Adaptación de estrategias a grupos etarios específicos

Prevención Secundaria

Uso efectivo de servicios online Asesoría y consejería por medios no presenciales
Mitigación de efectos de aislamiento Manejo del tiempo Manejo de estrés en relaciones

Prevención VIF Adaptación de estrategias a grupos etáreos específicos
Prevención terciaria 


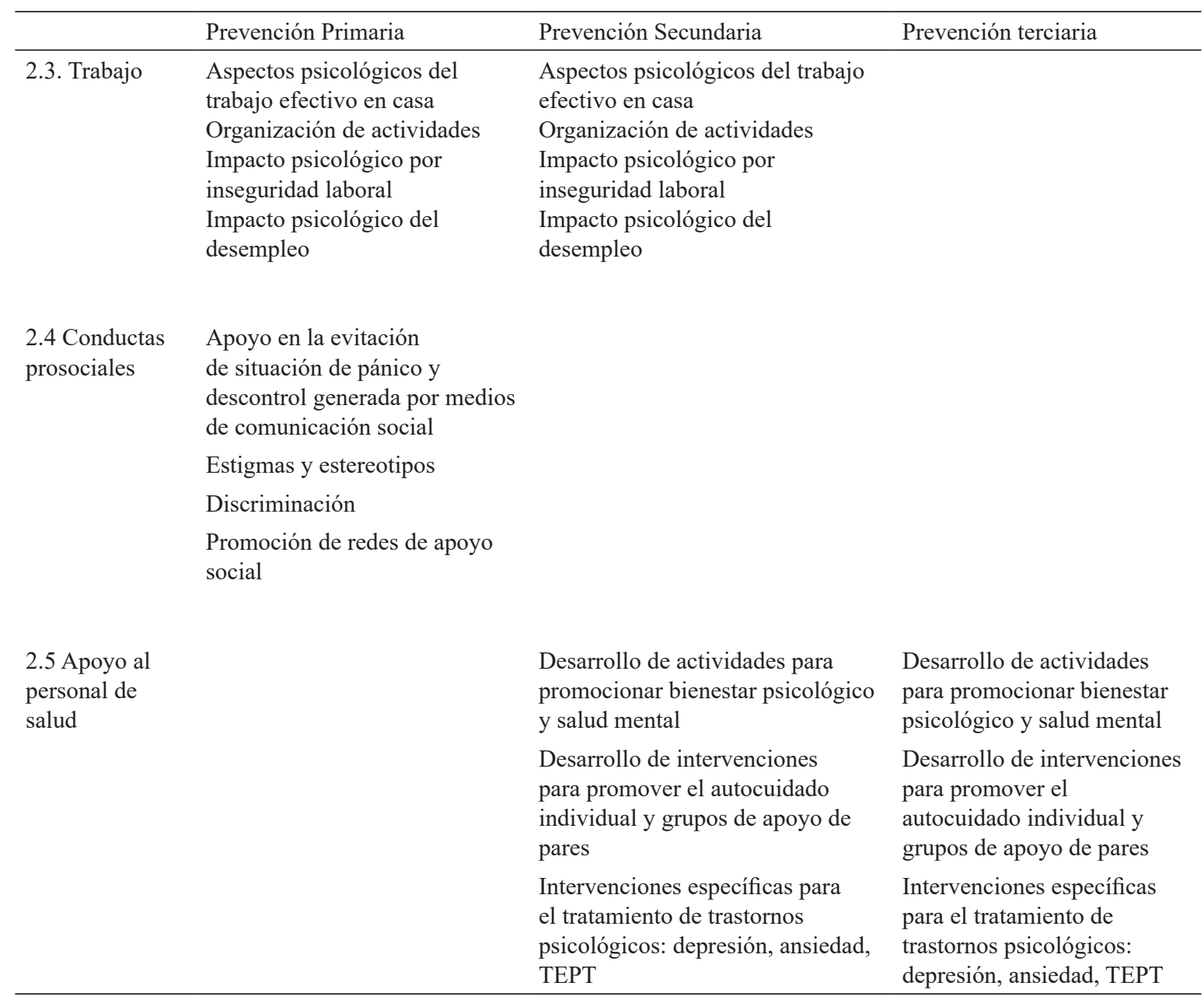

Las intervenciones han cobrado prioridad en diversos países. El gobierno de China ya principios rectores de la intervención de emergencia en crisis psicológicas para neumonía con nueva infección por coronavirus (National Health Comission of China, 2020) donde plantean como principios básicos (1) Incluir la intervención de crisis psicológica en el despliegue general de prevención y control de epidemias, con la premisa de reducir el daño psicológico causado por la epidemia y promover la estabilidad social, y ajustar oportunamente el enfoque de la intervención de crisis psicológicas de acuerdo con el progreso de la prevención y el control de epidemias, y (2) Implemente intervenciones clasificadas contra diferentes grupos de personas para proteger estrictamente la privacidad personal de los destinatarios. Tanto los implementadores como los receptores deben tener cuidado para evitar el trauma. En dicho documento plantean una serie de acciones tendientes a prestar servicios de salud mental para las personas afectadas, así como intervención de crisis psicológica para las personas que lo necesiten, prevenir, mitigar y tratar de controlar activamente el impacto psicosocial de la epidemia y por último mantener eficazmente el manejo y tratamiento de los trastornos mentales graves. Las intervenciones propuestas pueden ser revisadas en el anexo 1.

\section{Un ejemplo de modelo de intervención en marcha}

Considerando que gran parte de la intervención para tratar el COVID-19 incluyen aislamiento y cuarentena, se ha puesto en jaque las intervenciones psicológicas tradicionales cara a cara. Es por esto por lo que especialistas han desarrollado un modelo de intervención para abordar los problemas psicológicos urgentes 
de las personas (Fig. 2). Zhang, Wu, Zhao \& Zhang (2020) informan de un nuevo modelo de intervención de crisis psicológica utilizando tecnología de Internet. Este nuevo modelo, uno de West China Hospital, integra a médicos, psiquiatras, psicólogos y trabajadores sociales en plataformas de Internet para llevar a cabo una intervención psicológica a los pacientes, sus familias y el personal médico. Esta intervención utiliza como soporte la tecnología de Internet (redes $4 \mathrm{G}$ o $5 \mathrm{G}$ y teléfonos inteligentes).

El supuesto de base, afirman los autores, es que la intervención de crisis psicológica debe ser dinámica, para adaptarse fácilmente a las diferentes etapas de la epidemia (durante y después del brote). Durante el brote se plantea que la intervención psicológica en crisis debe abordar dos cuestiones

a. intervención por miedo a la enfermedad, realizada por médicos y psicólogos

b. intervención por dificultad en la adaptación, principalmente realizada por psicólogos. En caso de existir problemas mentales graves como violencia o conductas suicidas son manejadas por psiquiatras

1. Entrenamiento y Educación para la Salud

2. Intervención en crisis para personas de alto riesgo

3. Servicio Online $24 / 7$

4. Apoyo Social Online

Fig. 2. Modelo de intervención de crisis psicológica Zhang, Wu, Zhao \& Zhang (2020)

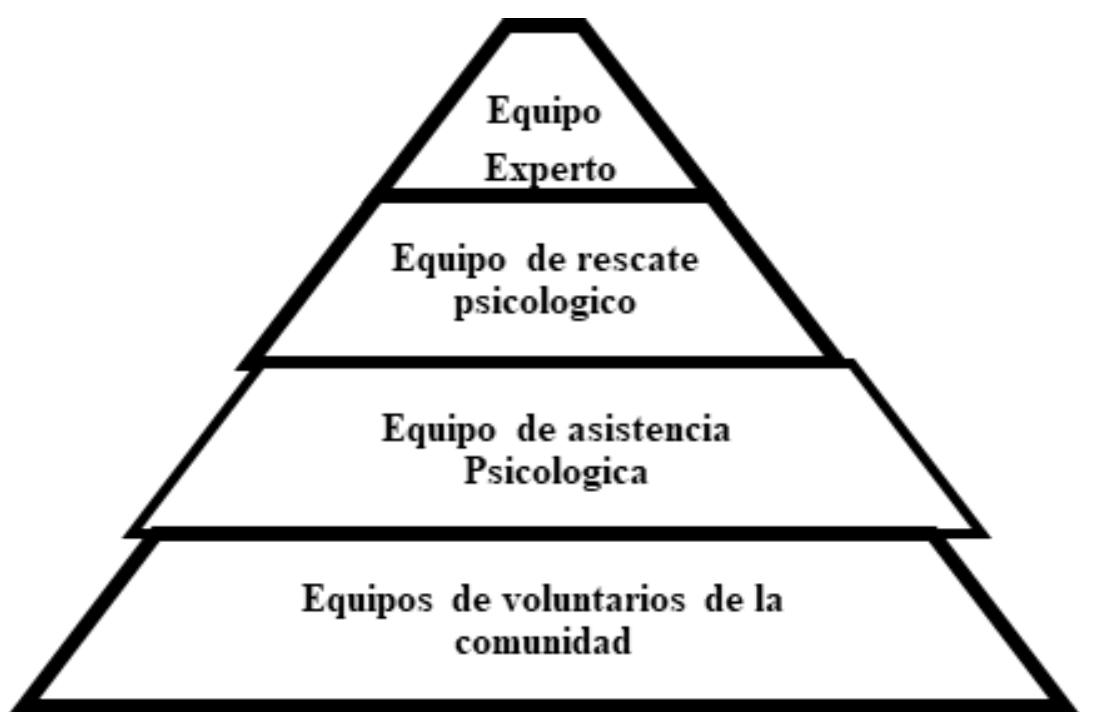

Adaptado de Zhang, Wu, Zhao \& Zhang (2020)

Figura 3. Modelo West China Hospital de atención psicológica integral

El modelo de atencion se observa en la figura 3. Como se observa existen cuatro grupos de asistentes e interventores psicológicos y todos mediados con el uso de tecnologias de internet. En una primera base se encuentra las comunidades, que principalmente brindan apoyo psicosocial. La asistencia psicológica en línea busca poder identificar y ayudar a los grupos objetivo que necesitan intervención. Por medio de tres aplicaciones de internet, Huayitong y el App, línea telefónica directa y la plataforma WeChat se organizan en forma rápida a médicos en todos los niveles del Hospital de China Occidental y psicólogos de toda la provincia de Sichuan para formar equipos de rescate psicológico. 


\section{¿Por qué avanzo tan rápido la enfermedad?}

Un hecho altamente llamativo en esta enfermedad es la paradoja que a pesar de no ser una enfermedad fulminante si ha sido rápidamente propagada a nivel mundial y particularmente en algunos países como Italia su propagación así de una velocidad alarmante. Diversas son las causas que han podido incidir en que parte de la población no adopte las conductas preventivas, entre estas una baja percepción de riesgo dada por un optimismo ilusorio.

Los estudios sobre optimismo y salud han identificado dos tipos de optimismo. El realista y el ilusorio (Torres-Salazar, Moreta-Herrera, Ramos-Ramírez. \& López-Castro, 2019: López \& Leffingwell, 2020). El primero se sustenta en esperar cosas positivas que ocurrirán, pero con un criterio anclado a la evidencia y hechos. Mientras el optimismo ilusorio lleva a que los sujetos esperen cosas positivas casi mágicamente independiente de lo que ellos hagan. Este último caso se ha identificado como riesgoso para la salud, ya que podría llevar a que sujetos con esa tendencia pseudo optimista e ilusoria realicen incluso comportamientos riesgosos para su salud ya que esperan que ellos no se enfermaran.

A fines de febrero de 2020 se llevó a cabo una encuesta en cuatro países de Europa que incluyeron a Francia, Italia, Reino Unido y Suiza $(\mathrm{N}=4,348)$ (Raude et al., 2020). Se evaluó las percepciones públicas del riesgo de infección por coronavirus pidiéndoles a los participantes que estimaran las probabilidades de contraer la enfermedad en los próximos meses. Dentro de los principales resultados se descubrió que las personas estimaron el riesgo de coronavirus de manera optimista. Es decir, el riesgo percibido de infección para uno mismo como el de otros fue muchísimo más bajo de lo esperado. Además, aproximadamente la mitad de los encuestados estimo la infección era menos probable que les ocurriera a ellos que a la población general. En opinión de los autores concluían que la mayoría de las personas en Europa estaban más sujetas a un optimismo "poco realista" sobre el riesgo de infección por coronavirus. Lamentablemente semanas después se ha visto que la epidemia avanzo muy rápidamente fuera de todo lo esperado por Europa y especialmente en Italia.

Por otra parte, un proceso básico en Psicología (y por lo tanto completamente sustentado en evidencia experimental) ayuda a explicar y complementar lo planteado anteriormente.

Tal como lo planteaban Bayes y Ribes (Bayés \& Ribes, 1992) un aspecto presente y no siempre considerado es la variable contingencia en el proceso del comportamiento y Salud. Como es sabido, la contingencia es la variable presente entre la emisión de una determinada conducta y la llegada de las consecuencias. Este fenómeno explica si un determinado comportamiento se adquiere, mantiene o se elimina o incluso si es más resistente a la extinción. Muchos de los problemas graves en salud o difícil de intervenir-prevenir, por ejemplo, adicciones o enfermedades de transmisión sexual son posibles de entender por la contingencia. En pocas palabras cuando los sujetos percibimos que entre la emisión de una determinada conducta y la "eventual" llegada de las consecuencias (sean positivas o negativas para el) es poco probable o pasa mucho tiempo este comportamiento es más difícil de adquirir o modificar. Esto llevaría por ejemplo a problemas serios de adherencia cuando un sujeto debe cambiar su alimentación por un problema serio de salud o para mantenerse sano. El postergar un alimento rico pero dañino en el momento es más difícil de cambiar si el posible beneficio se verá solo meses después.

Este fenómeno y proceso psicológico estaría presente también en la epidemia COVID-19. Si revisamos la evidencia hasta este momento, la enfermedad se ha vuelto grave por la velocidad principalmente de propagación (a pesar de ser potencialmente "fácil" de prevenir) por todo el mundo. ¿Cómo se explicaría que a pesar de información, educación y medidas rápidamente informadas por los gobiernos e instituciones de salud esto haya ocurrido?

Un segundo dato hasta este momento es la investigación descrita anteriormente (Raude et al 2020) donde se detectó que en promedio ciudadanos europeos estimaron su probabilidad de riesgo en forma optimista siendo en este caso una estimación (percepción, y creencia, dos fenómenos básicos nuevamente en psicología) riesgosa y como vemos llevó a las consecuencias hasta este momento que hemos lamentablemente experimentado. Esto traducido en los procesos básicos de adquisición de comportamiento y contingencia es que probablemente los sujetos no consideraron las medidas, indicaciones y riesgo de seguir con determinadas conductas y la necesidad de alterar y adecuar su estilo de vida precisamente porque no percibían el riesgo inmediato, solo una eventual posibilidad a futuro y que lo más probable no le ocurriera a él si no a otros. Esto fue lo ya detectado en la investigación, y la explicación de esa percepción puede ser el tipo de contingencia percibido por la población ante las campañas de prevención masiva. 
Entonces un modelo explicativo hipotético de la causa de esta pandemia y su propagación alarmante puede ser vista hasta este momento incorporando la percepción optimista ilusoria y el tipo de contingencia entre la información sobre la enfermedad -cambio de conductas y llegada de sus consecuencias.

\section{Comentarios Finales}

El COVID -19 nos ha mostrado que, aunque el factor biológico es esencial (virus, mecanismos biológicos involucrados y tratamiento farmacológico u otros disponibles), los factores psicológicos y sociales lo han transformado en un desastre mundial. El rápido avance y el impacto de la enfermedad en diversos ámbitos de la vida de las personas, las familias, las comunidades y la sociedad en general ha generado la urgente necesidad de que la ciencia psicológica y todas las otras disciplinas científicas consideren toda la evidencia disponible para afrontar una crisis sanitaria a nivel mundial, dado que todos los grandes desastres de la naturaleza y salud implican variables y/o consecuencias psicológicas importantes que deben ser consideradas (Vera-Villarroel \& Vogel, 2010; Vera-Villarroel, Celis-Atenas, Córdova-Rubio, Zych \& Buela-Casal, 2011).

Para la psicología, el desafío es recuperar la evidencia científica que por décadas han generado sus actores para incorporar en forma clara, precisa toda la evidencia científica disponible para la contribución en los problemas y desastres en salud en este caso.

Como se describió el gran problema de esta enfermedad ha estado vinculado al comportamiento de los sujetos, la necesidad de cambios en nuestro estilo de vida habitual. Este comportamiento que está estrechamente ligado a nuestras cogniciones, emociones y factores sociales y culturales deben ser incorporados en los análisis que se hagan a futuro, en las campañas de prevención y en la promoción de la Salud ya que, a pesar del poco tiempo transcurrido, ya contamos con información sobre los procesos psicológicos involucrados en el COVID-19 y con evidencia disponible que explica todos estos fenómenos, la cual debe ser puesta en relieve por los mismos actores de la psicología a disposición de otras disciplinas y sobre todo para los tomadores de decisión.

Se debe incorporar los principios psicológicos ya conocidos y avalados por décadas de investigación en las campañas de prevención, en los medios de comunicación, en la capacitación en los líderes políticos y de opinión (Garfin, Silver \& Holman, 2020). Así como también se requiere que este conocimiento psicológico pueda rápidamente incorporarse a las nuevas tecnologías. La experiencia descrita anteriormente ya puesta en marcha en este desastre pero que fue iniciada por otros desastres específicamente terremotos muestra que estas aplicaciones y el uso de teléfonos inteligentes e internet es una buena vía para realizar intervenciones a la población. Sin embargo, esta tecnología debe contener y desarrollarse a partir de la evidencia científica y no solo la construcción de tecnologías sin sentido social y sin base científica para la Salud.

La psicología y especialmente la psicología de la Salud cuenta con mucha evidencia y desarrollos y como hemos intentado acá de mostrar este conocimiento está a la base del gran problema sanitario que estamos enfrentados.

\section{Referencias}

Bayés, R., \& Ribes, E. (1992). Un modelo psicológico de prevención de enfermedad y su aplicación al caso del SIDA. En Piña, J.A. (Comp.), Psicología y salud: aportes del análisis de la conducta (pp. 1-21). Hermosillo, México: Editorial UNISON.

Brooks, S., Webster, R., Smith, L., Woodland, L., Wessely S., Greenberg, N., \& Rubin, G.J. (2020). The psychological impact of quarantine and how to reduce it: rapid review of the evidence. Lancet, 395, $912-920$.

Dai, Y., Hu, G., Xiong, H., Qiu, H., \& Yuan, X. (2020). Psychological impact of the coronavirus disease 2019 (COVID-19) outbreak on healthcare workers in China. MedRxiv (preprint). doi: 10.1101/2020.03.03.20030874

Garfin, D. R., Silver, R. C., \& Holman, E. A. (2020, March 23). The Novel Coronavirus (COVID-2019) Outbreak: Amplification of Public Health Consequences by Media Exposure. Health Psychology Journal (Advance online publication). doi:10.1037/hea0000875

Guo, Y.R., Cao, Q.D., Hong, Z.S., Tan Y.Y., Chen, S.D., Jin, H.J. (2020) The origin, transmission and clinical therapies on coronavirus disease 2019 (COVID-19) outbreak - an update on the status. Military Medical Research, 7, 11. doi: 10.1186/s40779-020-00240-0.

Jeong, H., Woo, H., Song, Y. J., Ki, M., Min, J., Cho, J., \& Chae, J,H. (2016). Mental health status of people isolated due to Middle East 
Respiratory Syndrome. Epidemiology and Health, 38: e2016048. doi: https://doi.org/10.4178/epih.e2016048

Jiang, X., Deng, L., Zhu, Y., Ji, H., Tao, L., Liu, L., \& Yang, D., Ji, W. (2020) Psychological crisis intervention during the outbreak period of new coronavirus pneumonia from experience in Shangai. Psychiatry Research, 286, 112903. doi:10:1016/j.psychres.2020.112903.

Lee, A.M., Wong, J., McAlonan, G.M., Cheung, V., Cheung, C.C, Sham, P., Chu, C., Wong, P., Tsang, K., \& Chua, S.A, (2007). Stress and Psychological Distress Among SARS Survivors 1 Year After the Outbreak. The Canadian Journal of Psychiatry, 52 (4), 233-240.

Li, S., Wan, Y., Xue, J., Zhao, N., \& Zhu, T. (2020). The Impact of COVID-19 Epidemic Declaration on Psychological Consequences: A Study on Active Weibo Users. International Journal of Environmental Research and Public Health, 17(6), 2032. doi:10.3390/ ijerph17062032

Lopez, S. V., \& Leffingwell, T. R. (2020) The Role of Unrealistic Optimism in College Student Risky Sexual Behavior, American Journal of Sexuality Education. (Advance online publication) doi: 10.1080/15546128.2020.1734131

Lunn, P. D., Belton, C. A., Lavin, C., McGowan, F. P., Timmons, S., \& Robertson, D. A. (2020). Using Behavioral Science to help fight the Coronavirus. Journal of Behavioral Public Administration, 3(1). https://doi.org/10.30636/jbpa.31.147

Main, A., Zhou, Q., Ma, Y., Luecken, L., \& Liu, X. (2011). Relations of SARS-Related Stressors and Coping to Chinese College Students' Psychological Adjustment During the 2003 Beijing SARS Epidemic. Journal of Counseling Psychology, 58, 1410-1423. doi: 10 IO37/aOO23632

Murphy, D. J. (2020). Framework for contribution of Psychology \& Psychologist to COVID-19 Pandemic. Recuperado desde https:// twitter.com/ClinPsych David/status/1242891161951514629

National Health Commission of China. (2020) Principles of the emergency psychological crisis interventions for the new coronavirus pneumonia [en Chino]. Recuperado desde http://www.nhc.gov.cn/jkj/s3577/202001/6adc08b966594253b2b 791be5c3b9467External Link

Parka, J., Leeb, E., Parkc, N., \& Choid, Y. (2018). Mental Health of Nurses Working at a Government-designated Hospital During a MERS-CoV Outbreak: A Cross-sectional Study. Archives of Psychiatric Nursing, 32, 2-6.

Poudel Adhikari, S., Meng, S., Wu, Y., Mao, Y., Ye, R., Wang, Q., Sun, C., Sylvia, S., Rozelle, S., Raat, H., \& Zhou, H. (2020). A Literature Review of 2019 Novel Coronavirus During the Early Outbreak Period: Epidemiology, Causes, Clinical Manifestation and Diagnosis, Prevention and Control. Preprints. doi: 10.20944/preprints202002.0060.v1.

Raude, J., Debin, M., Souty, C., Guerrisi, C., Turbelin, C., Falchi, A., ... Colizza, V. (2020, March 8). Are people excessively pessimistic about the risk of coronavirus infection?. https://doi.org/10.31234/osf.io/364qj.

She, K., Yanq, Y., Wang, T., Zhao, D., Jianq, Y., ...Jin, R. (2020). Diagnosis, treatment, and prevention of 2019 novel coronavirus infection in children: experts consensus statement. World Journal of Pediatrics. (Advance online publication). doi: 10.1007/s12519-020-00343-7.

Shigemura, J., Ursano, R. J., Morganstein, J. C., Kurosawa, M., \& Benedek, D. M. (2020). Public responses to the novel 2019 coronavirus (2019-nCoV) in Japan: Mental health consequences and target populations. Psychiatry and Clinical Neurosciences. (Advance online publication). doi: 10.1111/pcn.12988

Torales, J., O’Higgins, M., Castaldelli-Maia, J. M., \& Ventriglio, A. (2020). The outbreak of COVID-19 coronavirus and its impact on global mental health. International Journal of Social Psychiatry, 31. (Advance online publication) doi:10.1177/0020764020915212

Torres-Salazar, C., Moreta-Herrera, R., Ramos-Ramírez, M., \& López-Castro, J. (2019). Sesgo Cognitivo de Optimismo y Percepción de Bienestar en una muestra de Universitarios Ecuatorianos. Revista Colombiana de Psicología, 29, 61-72 doi: 10.15446/rcp.v29n1.75853

Vera-Villarroel, P., Celis-Atenas, K., Córdova-Rubio, N., Zych, I., \& Buela-Casal, G. (2011). Chilean Validation of the Posttraumatic Stress Disorder Checklist-Civilian Version (PCL-C) after the Earthquake on February 27, 2010. Psychological Reports, 109, 47-58. doi:10.2466/02.13.15.17.PR0.109.4.47-58

Vera-Villarroel, P., Livacic-Rojas, P., Fernández, A. M., \& Oblitas, L. (2015). Principales modelos teóricos en psicología de la salud. En Vera-Villarroel, P., \& Oblitas, L-A. (Dir). Manual de Escalas y Cuestionarios Iberoamericanos en Psicologia Clinica y de la Salud. PSICOM: editores.

Vera-Villarroel, P., \& Vogel,EH. (2010). Psychology and natural disasters: earthquake and tsunami in Chile on February $27,2010$. Terapia Psicológica, 28, 143-145.

Wang, C., Pan, R., Wan, X., Tan, Y., Xu, L., Ho, C.S., \& Ho, R.C. (2020). Immediate Psychological Responses and Associated Factors during the Initial Stage of the 2019 Coronavirus Disease (COVID-19) Epidemic among the General Population in China. International Journal of Environmental Research and Public Health, 17, 1729. doi:10.3390/ijerph17051729.

Yang, S., \& Cho, S. (2017). Middle East respiratory syndrome risk perception among students at a university in South Korea, 2015. American Journal of Infection Control, 45, e53-e60. doi: https://doi.org/10.1016/j.ajic.2017.02.013

Zaki, A.M., van Boheemen, S., Bestebroer, T.M., Osterhaus, A.D., \& Fouchier, R.A. (2012). Isolation of a novel coronavirus from a man with pneumonia in Saudi Arabia. The New England Journal of Medicine, 367, 1814-1820. doi: 10.1056/nejmoa1211721.

Zhang, J., Wu, W., Zhao, X., \& Zhang, W. (2020). Recommended psychological crisis intervention response to the 2019 novel coronavirus pneumonia outbreak in China: a model of West China Hospital. Precision Clinical Medicine, 00, 1-6 doi: 10.1093/pcmedi/ 
pbaa006 Perspective

Zhu, Y., Chen, L., Ji, H., Xi, M., Fang, Y., \& Li, Y. (2020) The risk and prevention of novel coronavirus pneumonia infections among inpatiens in psychiatric hospitals. Neuroscience Bulletin. (Advance online publication). doi: 10.1007/s12264-020-00476-9.

\section{Anexo 1}

Principales medidas de intervención en crisis psicológica para diferentes grupos propuestas por la Comisión Nacional de Salud de China (Adaptado de National Health Comission, 2020)

\begin{tabular}{|c|c|c|}
\hline $\begin{array}{l}\text { Pacientes diag- } \\
\text { nosticados en } \\
\text { etapa temprana } \\
\text { del tratamiento } \\
\text { de aislamiento }\end{array}$ & $\begin{array}{l}\text { Mentalidad: entumecimien- } \\
\text { to, negación, ira, miedo, } \\
\text { ansiedad, depresión, des- } \\
\text { ilusión, quejas, insomnio o } \\
\text { agresión, etc. } \\
\text { Principio: Soporte y co- } \\
\text { modidad. Trate al paciente } \\
\text { con tolerancia, estabilice } \\
\text { las emociones del paciente } \\
\text { y evalúe el riesgo de sui- } \\
\text { cidio, autolesión y ataque } \\
\text { temprano. }\end{array}$ & $\begin{array}{l}\text { Comprenda que la respuesta emocional del paciente es una res- } \\
\text { puesta normal al estrés, esté preparado de antemano y no se enfade } \\
\text { por la agresión y el comportamiento triste del paciente y pierda la } \\
\text { posición del médico, como las disputas con el paciente o una parti- } \\
\text { cipación excesiva. } \\
\text { Bajo la premisa de comprender al paciente, además del tratamiento } \\
\text { farmacológico, se debe administrar una intervención psicológica en } \\
\text { crisis, como la evaluación oportuna del suicidio, las autolesiones, el } \\
\text { riesgo de ataque, el apoyo psicológico positivo y ningún conflicto } \\
\text { positivo con el paciente. Consulte una consulta psiquiátrica si es } \\
\text { necesario. Explicar la importancia y la necesidad de la terapia de } \\
\text { aislamiento y alentar a los pacientes a generar confianza en la recu- } \\
\text { peración activa. } \\
\text { Haga hincapié en que el método de aislamiento no es solo para } \\
\text { observar y tratar mejor a los pacientes, sino también para proteger } \\
\text { a los miembros de la familia y la seguridad social. Explicar los } \\
\text { puntos principales del tratamiento actual y la efectividad de la in- } \\
\text { tervención. }\end{array}$ \\
\hline $\begin{array}{l}\text { Pacientes } \\
\text { diagnosticados } \\
\text { en período de } \\
\text { tratamiento y en } \\
\text { aislamiento }\end{array}$ & $\begin{array}{l}\text { Mentalidad: además de lo } \\
\text { anterior, puede haber sole- } \\
\text { dad o no cooperar debido } \\
\text { al miedo a la enfermedad, } \\
\text { abandonar el tratamiento } \\
\text { u optimismo excesivo y } \\
\text { expectativas excesivas de } \\
\text { tratamiento } \\
\text { Principios: Comunique } \\
\text { activamente información y } \\
\text { consulte con psiquiátricos } \\
\text { cuando sea necesario. }\end{array}$ & $\begin{array}{l}\text { Según el grado aceptable del paciente, explique objetiva y veraz- } \\
\text { mente la enfermedad y la situación epidémica externa, para que el } \\
\text { paciente conozca el corazón. } \\
\text { Ayude a comunicarse con familiares externos y transmita informa- } \\
\text { ción. } \\
\text { Anime activamente al paciente a cooperar con todas las acciones de } \\
\text { tratamiento. } \\
\text { Intente hacer que el ambiente sea adecuado para el tratamiento de } \\
\text { los pacientes. }\end{array}$ \\
\hline
\end{tabular}




\begin{tabular}{|c|c|c|}
\hline $\begin{array}{l}\text { Pacientes } \\
\text { diagnosticados } \\
\text { con dificultad } \\
\text { respiratoria, an- } \\
\text { siedad extrema } \\
\text { y dificultad para } \\
\text { expresarse }\end{array}$ & $\begin{array}{l}\text { Mentalidad: cerca de la } \\
\text { muerte, pánico, desespera- } \\
\text { ción, etc. } \\
\text { Principios: Calmar, cal- } \\
\text { mar, prestar atención a la } \\
\text { comunicación emocional y } \\
\text { mejorar la confianza en el } \\
\text { tratamiento. }\end{array}$ & $\begin{array}{l}\text { Mientras calma y apacigua, fortalece el tratamiento de la enferme- } \\
\text { dad primaria y alivia los síntomas }\end{array}$ \\
\hline $\begin{array}{l}\text { Pacientes diag- } \\
\text { nosticados con } \\
\text { síntomas leves } \\
\text { aislados en el } \\
\text { hogar y pacien- } \\
\text { tes con fiebre } \\
\text { que fueron al } \\
\text { hospital para } \\
\text { recibir trata- } \\
\text { miento. }\end{array}$ & $\begin{array}{l}\text { Mentalidad: pánico, inquie- } \\
\text { tud, soledad, impotencia, } \\
\text { depresión, depresión, pe- } \\
\text { simismo, ira, nerviosismo, } \\
\text { estrés de distanciarse de los } \\
\text { demás, agravio, vergüenza } \\
\text { o desprecio por enfermeda- } \\
\text { des, etc } \\
\text { Principio: Educación para } \\
\text { la salud, fomentar la coo- } \\
\text { peración y adaptarse a los } \\
\text { cambios. }\end{array}$ & $\begin{array}{l}\text { Ayudar a los pacientes a comprender información y conocimiento } \\
\text { reales y confiables, y obtener materiales científicos y médicos au- } \\
\text { torizados. } \\
\text { Fomentar la cooperación activa con medidas de tratamiento y } \\
\text { aislamiento, dieta saludable, trabajo y descanso, leer más libros, } \\
\text { escuchar música, usar métodos modernos de comunicación y otras } \\
\text { rutinas diarias. Actividades. } \\
\text { Acepte el aislamiento, comprenda sus reacciones y busque un sig- } \\
\text { nificado positivo en la adversidad. } \\
\text { Busque apoyo social para lidiar con el estrés: use métodos moder- } \\
\text { nos de comunicación para contactar a familiares, amigos, colegas, } \\
\text { etc., hable sobre sentimientos, mantenga la comunicación con la } \\
\text { sociedad y obtenga apoyar y alentar. } \\
\text { Fomentar el uso de la línea directa de asistencia o intervención psi- } \\
\text { cológicas en línea. }\end{array}$ \\
\hline $\begin{array}{l}\text { Personas con } \\
\text { sospecha de in- } \\
\text { fección }\end{array}$ & $\begin{array}{l}\text { Mentalidad: casualidad, } \\
\text { evitar el tratamiento, miedo } \\
\text { a ser discriminado o ansie- } \\
\text { dad, tratamiento excesivo, } \\
\text { traslados frecuentes al hos- } \\
\text { pital, etc. } \\
\text { Principios: Propaganda y } \\
\text { educación de manera opor- } \\
\text { tuna, protección correcta, } \\
\text { obedecer la situación gene- } \\
\text { ral y reducir el estrés. }\end{array}$ & $\begin{array}{l}\text { Misiones políticas, observación cercana y tratamiento temprano. } \\
\text { Adoptar las medidas de protección necesarias para usted. } \\
\text { Obedecer el arreglo general e informar las condiciones personales } \\
\text { de acuerdo con las regulaciones. } \\
\text { Usar comportamientos de descompresión y reducir el estrés. }\end{array}$ \\
\hline
\end{tabular}




\begin{tabular}{|c|c|c|}
\hline $\begin{array}{l}\text { Personal médi- } \\
\text { co y equipo de } \\
\text { salud }\end{array}$ & $\begin{array}{l}\text { Mentalidad: fatiga y ten- } \\
\text { sión excesivas, incluso } \\
\text { agotamiento, ansiedad, } \\
\text { insomnio, depresión, tris- } \\
\text { teza, agravios, impotencia, } \\
\text { depresión, frustración o } \\
\text { autoculpa frente a la muerte } \\
\text { del paciente. Preocuparse } \\
\text { por estar infectado, preo- } \\
\text { cuparse por los miembros } \\
\text { de la familia, temer que los } \\
\text { miembros de la familia se } \\
\text { preocupen por ellos mis- } \\
\text { mos. Emoción excesiva, } \\
\text { rechazar un descanso razo- } \\
\text { nable y no poder garantizar } \\
\text { su salud. } \\
\text { Principio: rotación regular, } \\
\text { autorregulación y buscar } \\
\text { ayuda si tiene alguna pre- } \\
\text { gunta. }\end{array}$ & $\begin{array}{l}\text { Realice un entrenamiento de intervención psicológica en crisis } \\
\text { antes de participar en el rescate, comprenda la respuesta al estrés } \\
\text { y aprenda a lidiar con el estrés y regular las emociones. Realice } \\
\text { entrevistas preventivas y discuta abiertamente los sentimientos in- } \\
\text { ternos; apoyo y comodidad; movilización de recursos; y ayude a las } \\
\text { partes a prepararse psicológicamente para el estrés. } \\
\text { Elimine las preocupaciones de los trabajadores médicos de primera } \\
\text { línea, haga arreglos para que personal dedicado brinde apoyo logís- } \\
\text { tico e intente rotar al personal en el área de aislamiento una vez al } \\
\text { mes. } \\
\text { Programación razonable, organice la relajación y el descanso apro- } \\
\text { piados, garantice un sueño y una dieta adecuados. Intente organizar } \\
\text { el personal de primera línea del hospital designado para que se que- } \\
\text { de cerca del hospital. } \\
\text { Cuando sea posible, manténgase en contacto y comuníquese con su } \\
\text { familia y el mundo exterior. } \\
\text { En caso de insomnio, bajo estado de ánimo, ansiedad, puede buscar } \\
\text { intervención profesional en crisis psicológica o servicios de salud } \\
\text { mental, puede llamar a la línea directa de asistencia psicológica o } \\
\text { servicios psicológicos en línea, y la intervención psicológica en } \\
\text { persona puede llevarse a cabo en áreas calificadas. Si no se alivia } \\
\text { durante } 2 \text { semanas y afecta a los trabajadores, debe ser evaluado y } \\
\text { tratado por el departamento de psiquiatría. } \\
\text { Si se han producido síntomas de estrés, debe ajustar su puesto de } \\
\text { trabajo a tiempo y buscar ayuda profesional. }\end{array}$ \\
\hline $\begin{array}{l}\text { Personas que } \\
\text { están en contac- } \\
\text { to cercano con } \\
\text { el paciente (fa- } \\
\text { milia, colegas, } \\
\text { amigos, etc.) }\end{array}$ & $\begin{array}{l}\text { Mentalidad: evitación, in- } \\
\text { quietud, ansiedad durante } \\
\text { el período de espera, o } \\
\text { coraje ciego, rechazo a la } \\
\text { protección y observación en } \\
\text { el hogar } \\
\text { Principios: misión, co- } \\
\text { modidad y fomento de la } \\
\text { comunicación a través de } \\
\text { Internet. }\end{array}$ & $\begin{array}{l}\text { Propaganda de políticas, estímulo para enfrentar la realidad, } \\
\text { cooperar con la observación en el hogar. } \\
\text { Difusión y comunicación de información adecuada, liberar la } \\
\text { tensión. }\end{array}$ \\
\hline $\begin{array}{l}\text { Personas que no } \\
\text { desean buscar } \\
\text { tratamiento } \\
\text { médico pública- } \\
\text { mente }\end{array}$ & $\begin{array}{l}\text { Mentalidad: miedo a un } \\
\text { diagnóstico erróneo y aisla- } \\
\text { miento, falta de conciencia, } \\
\text { evitación, negligencia, in- } \\
\text { quietud, etc. } \\
\text { Principio: explicar la per- } \\
\text { suasión, no criticar, apoyar } \\
\text { el comportamiento médico. }\end{array}$ & $\begin{array}{l}\text { Propaganda del conocimiento y eliminación del miedo. } \\
\text { Consulta temprana, beneficiar a otros; } \\
\text { Disipar la vergüenza, la protección científica; }\end{array}$ \\
\hline
\end{tabular}


Personas susceptibles y público en general.
Mentalidad: pánico, no se atreven a salir, desinfección a ciegas, desilusión, miedo, irritabilidad, comportamiento agresivo y exceso de optimismo, desistimiento, etc.

Principios: educación para la salud, orientación para la respuesta activa, eliminación del miedo y prevención científica.
Proporcione correctamente información e información sobre otros servicios.

Comunicación y orientación sobre el comportamiento adaptativo. No discrimine entre pacientes enfermos y sospechosos.

Recuerde la atención a los métodos de afrontamiento poco saludables (como beber alcohol, fumar, etc.).

Síntomas de autoidentificación. 